\title{
Active Filter Design Using Operational Transconductance Amplifiers: A Tutorial
}

\author{
Randall L. Geiger and Edgar Sánchez-Sinencio
}

\begin{abstract}
Basic properties of the Operational Transconductance Amplifier (OTA) are discussed. Applications of the OTA in voltage-controlled amplifiers, filters, and impedances are presented. A versatile family of voltage-controlled filter sections suitable for systematic design requirements is described. The total number of components used in these circuits is small, and the design equations and voltage-control characteristics are attractive. Limitations as well as practical considerations of OTA based filters using commercially available bipolar OTAs are discussed. Applications of OTAs in continuous-time monolithic filters are considered.
\end{abstract}

\section{Introduction}

The conventional operational amplifier (op amp) is used as the active device in the vast majority of the active filter literature. For design purposes, the assumption that the op amp is ideal $A_{v}=\infty, R_{\text {in }}=\infty, R_{0}=0$ is generally made, and large amounts of feedback are used to make the filter gain essentially independent of the gain of the op amp. A host of practical filter designs have evolved following this approach. It has also become apparent, however, that operational amplifier limitations preclude the use of these filters at high frequencies, attempts to integrate these filters have been unsuccessful (with the exception of a few nondemanding applications), and convenient voltage or current control schemes for externally adjusting the filter characteristics do not exist.

With the realization that the BJT and MOSFET are inherently current and transconductance amplifiers, respectively, the following question naturally arises. Can any improvements in filter characteristics, performance, or flexibility be obtained by using one of the other basic types of amplifiers (e.g., transconductance, current, or transresistance) in place of a voltage amplifier (or specifically the operational amplifier) as the basic active device in a filter structure?
This question is currently difficult to answer for two reasons. First, there is a near void in the literature of active filter structures employing the alternative amplifier types. Second, the evolution of good integrated transresistance, transconductance, and current amplifiers has not kept pace with that of the voltage amplifiers, although a few devices in these alternate categories are commercially available (e.g., transconductance amplifiers such as the CA 3080 and LM 13600 and transresistance amplifiers such as the LM 3900) [1]-[5]. Comparisons of some characteristics of these amplifiers were recently discussed by Brugger et al. [40].

In this paper, basic first- and second-order structures using the transconductance amplifier (often termed the operational transconductance amplifier: OTA) are discussed. It is shown that these structures offer improvements in design simplicity and programmability when compared to op amp based structures as well as reduced component count.

Many of the basic OTA based structures use only OTAs and capacitors and, hence, are attractive for integration. Component count of these structures is often very low (e.g., second-order biquadratic filters can be constructed with two OTAs and two capacitors) when compared to VCVS designs. Convenient internal or external voltage or current control of filter characteristics is attainable with these designs. They are attractive for frequency referenced (e.g., master/slave) applications. Several groups have recently utilized OTAs in continuous-time monolithic filter structures [28] - [40].

From a practical viewpoint, the high-frequency performance of discrete bipolar OTAs, such as the CA 3080, is quite good. The transconductance gain, $g_{m}$, can be varied over several decades by adjusting an external dc bias current, $I_{\mathrm{ABC}}$. The major limitation of existing OTAs is the restricted differential input voltage swing required to maintain linearity [5]. For the CA 3080, it is limited to about $30 \mathrm{mV}$ p-p to maintain a reasonable degree of linearity. Although feedback structures in which the sensitivity of the filter parameters are reduced (as is the goal in op amp based filter design) will be discussed, major emphasis will be placed upon those structures in which the standard filter parameters of interest are directly proportional to $g_{m}$ of the OTA. Thus, the $g_{m}$ will be a 
design parameter much as are resistors and capacitors. Since the transconductance gain of the OTA is assumed proportional to an external dc bias current, external control of the filter parameters via the bias current can be obtained.

Most existing work on OTA based filter design approached the problem by either concentrating upon applying feedback to make the filter characteristics independent of the transconductance gain or modifying existing op amp structures by the inclusion of some additional passive components and OTAS. In either case, the circuits were typically component intense and cumbersome to tune. Some of the earlier works are listed in the Refs. [6]-[16]. Some of the most practical circuits can be found in the manufacturer's application notes [3]-[5].

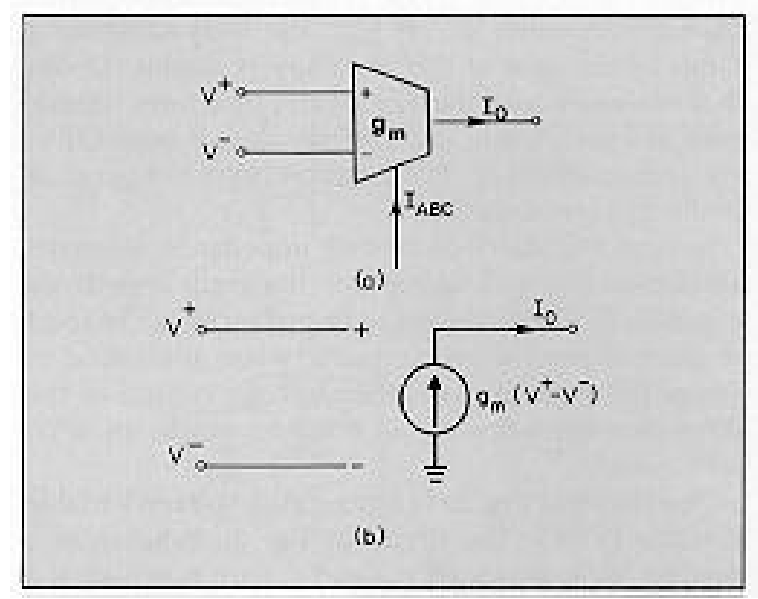

Fig.1 OTA. (a) Symbol. (b) Equivalent circuit of ideal OTA.

\section{OTA Model}

The symbol used for the OTA is shown in Fig. 1, along with the ideal small signal equivalent circuit. The transconductance gain, $g_{m}$, is assumed proportional* to $I_{\mathrm{ABC}}$. The proportionality constant $h$ is dependent upon temperature, device geometry, and the process [2].

$$
g_{m}=h I_{\mathrm{ABC}}
$$

\footnotetext{
* A linear dependence on bias current is typically obtained for bipolar OTAs and MOS configurations operating in weak inversion. MOS structures operating in the saturation region typically exhibit a quadratic dependence on $I_{\mathrm{ABC}}$
}

The output current is given by

$$
I_{o}=g_{m}\left(V^{+}-V\right)
$$

As shown in the model, the input and output impedances in the model assume ideal values of infinity. Current control of the transconductance gain can be directly obtained with control of IABC- Since techniques abound for creating a current proportional to a given voltage, voltage control of the OTA gain can also be attained through the IABC input. Throughout this paper, when reference is made to either the current or voltage controllability of OTA based circuits' it is assumed to be attained via control of $g_{m}$ by $I_{\mathrm{ABC}}$.

\section{Basic OTA Building Blocks}

Some of the basic OTA building blocks [6] are introduced in this section. A brief discussion about these circuits follows.

Voltage amplifiers using OTAs are shown in Fig. 2, along with voltage gain and output impedance expressions. The basic inverting and non-inverting configurations of Figs. 2a and $2 \mathrm{~b}$ have a voltage gain directly proportional to $g_{m}$, which makes current (voltage) control of the gain via $I_{\mathrm{ABC}}$ straightforward. Furthermore, observe that a differential amplifier can be easily obtained by using both input terminals of the OTA in Figs. $2 \mathrm{a}$ or $2 \mathrm{~b}$. The major limitation of these circuits is the relatively high output impedance.

A voltage buffer, such as used in Figs. $2 \mathrm{c}$ and $2 \mathrm{~d}$, is often useful for reducing output impedance.* Although the gain characteristics of these circuits are ideally identical, the performance of the two circuits is not the same. The performance differences are due to differences in the effects of parasitics in the circuits. Specifically, the parasitic output capacitance of the OTA in Fig. 2c, along with instrumentation parasitics, parallel the resistor $R_{L}$ in discrete component structures, thus causing a roll-off in the frequency response of the circuits. In the circuit of Fig. $2 d$, the parasitic output capacitance of the OTA is connected across the null port of an op amp and thus has negligible effects when the op amp functions properly. Likewise, instrumentation parasitics will typically appear at the low impedance output of the op amp, and thus not have a major effect on the performance. As with conventional amplifier design using resistors and op amp's, the amplifier bandwidth of these structures warrants consideration. For the circuits of Figs. 2c and 2d, the major factor limiting 


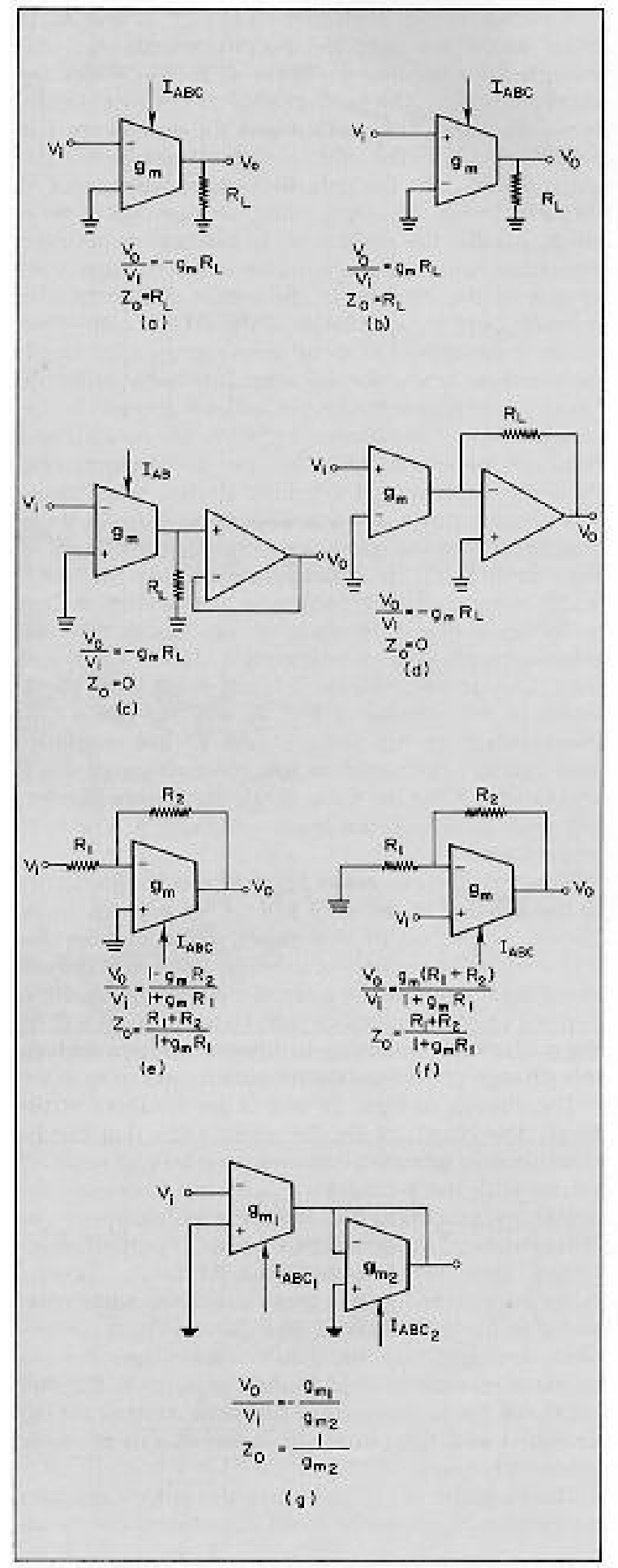

Fig. 2 Voltage amplifiers. (a) Basic inverting. (b) Basic noninverting. (c) Feedback amplifier. (d) Noninverting feedback amplifier. (3 Buffered amplifier. (f) Buffered VCVC feedback. (g) All OTA amplifiers. the bandwidth is generally the finite gain bandwidth product of the op amps. If the op amps are modeled by the popular single-pole roll-off model, $A(\mathrm{~s})=G B / s$, and the OTAs are assumed ideal, it follows that the bandwidth of the circuits of Fig. 2c and Fig. 2d is GB, independent of the voltage gain of the amplifier. This can be contrasted to the bandwidths of $G B / K$ and $G B / 1+K$ for the basic single op amp non-inverting and inverting amplifiers of gains $K$ and $K$, respectively.

Note that the circuits of Figs. 2a and 2b differ only in the labeling of the "+" and "-" terminals. In all circuits presented in this paper, interchanging the "+" and "'" terminals of the OTA will result only in changing the sign of the $g_{m}$ coefficient in any equation derived for the original circuit. Henceforth, it will be the reader's responsibility to determine when such an interchange provides a useful circuit.

The circuits of Figs. 2e and $2 \mathrm{f}$ are feedback structures. The circuit of Fig. 2e offers gains that can be continuously adjusted between positive and negative values with the parameter $g_{m}$. By interchanging the + and - terminals of the OTA, very large gains can be obtained as $g_{m} R_{1}$ approaches 1 (as $Z_{o}$ approaches infinity). Gain is nonlinearly related to $g_{m}$. Control range via $g_{m}$ is reduced in these structures when compared to the amplifiers of Figs. $2 a$ and $2 b$. If components are sized fitly, the gain of these structures can be made essentially independent of $g_{m}$ (as in the conventional op amp inverting and non-inverting configurations) and the output impedances can be made reasonably small.

The amplifier of Fig. $2 \mathrm{~g}$ is attractive since it contains no passive components. Gain adjustment can be attained with either $g_{m 1}$ or $g_{m 2}$. The total adjustment range of the gain of this structure is double (in $\mathrm{dB}$ ) that attainable with the single OTA structures considered in Figs. $2 a$ and $2 b$. Furthermore, if both OTAs are in the same chip, the variations with temperature of the $g_{m}$ 's are cancelled.

Several standard controlled impedance elements are shown in Fig. 3, along with the input impedance expression. These controlled impedances can be used in place of passive counterparts (when applicable) in active $R C$ structures to attain voltage control of the filter characteristics or as building blocks in OTA structures.

The circuit of Fig. 3a is a grounded Voltage Variable Resistor (VVR). The circuit of Fig. 3b behaves as a floating VVR, provided $g_{m 1}$ and $g_{m 2}$ are matched. If a mismatch occurs, the structure can be modeled with a floating VVR between terminals 1 and 2 of value $g_{m 1}$, along with a voltage dependent current source of value $\left(g_{m 1}-g_{m 2}\right) V_{1}$ driving node 2 . 


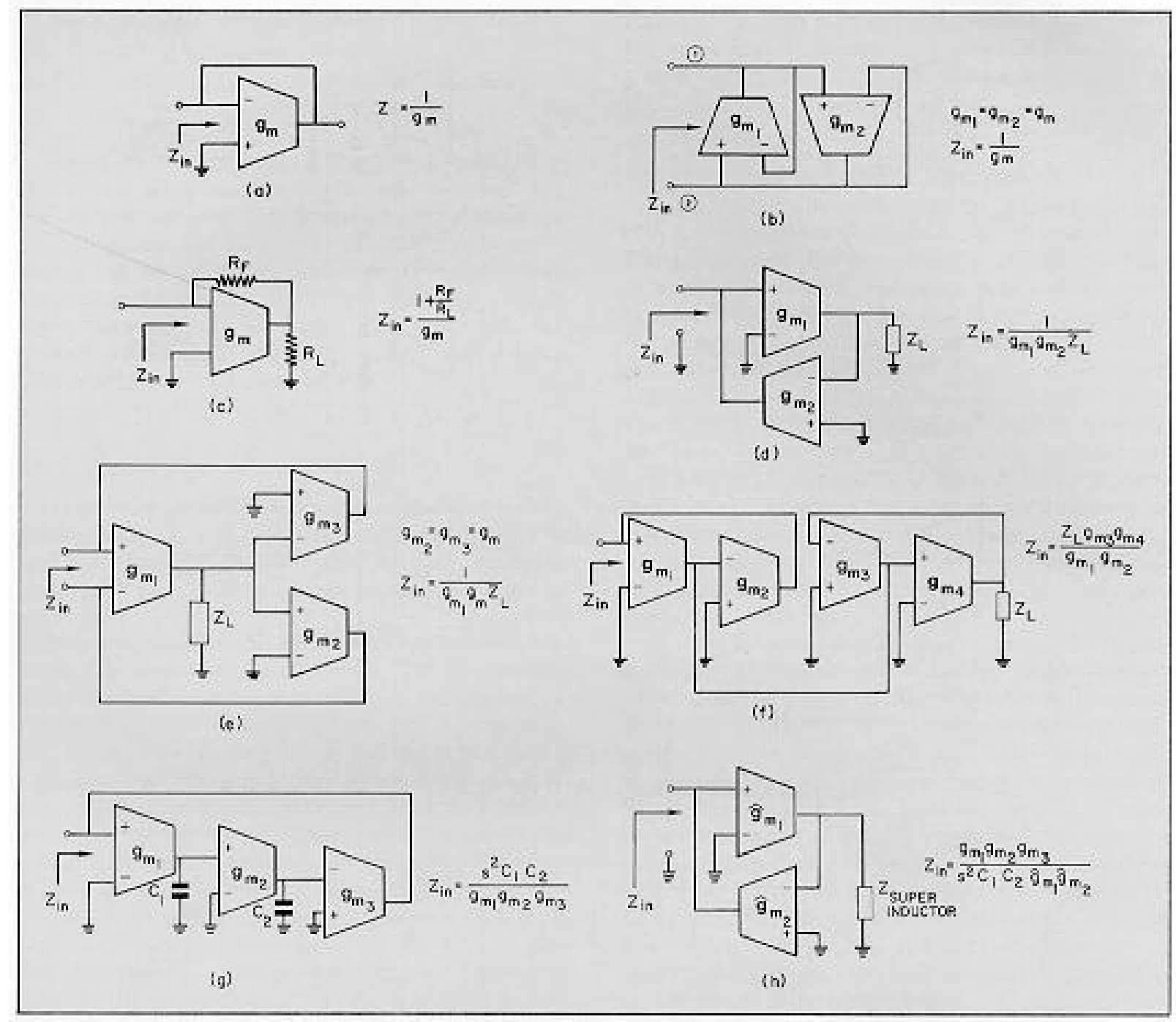

Fig. 3 Controlled impedance elements. (a) Single-ended voltage variable resistor (VVR). (b) Floating VVR. (c) Scaled VVR. (d) Voltage variable impedance inverter. (e) Voltage variable floating impedance. (f) Impedance multiplier. (g) Super inductor. ( $h$ ) FDNR.

The circuit of Fig. 3c acts as a scaled VVR. Higher impedances are possible than with the simple structure of Fig. 3a, at the expense of the additional resistors.

A voltage variable impedance inverter is shown in Fig. 3d. Note the doubling of the adjustment range of this circuit, as with the amplifier of Fig. 2g. Of special interest is the case where this circuit is loaded with a capacitor. In this case, a synthetic inductor is obtained. The doubling of the adjustment range is particularly attractive for the synthetic inductor since cutoff frequencies in active filter structures generally involve inductor values raised to the $1 / 2$ power. By making $g_{m 1}=g_{m 2}$ and adjusting both simultaneously, first-order rather than quadratic control of cutoff frequencies is possible.
A floating impedance inverter is shown in Fig. 3e. Note that it is necessary to match $g_{m 2}$ and $g_{m 3}$ for proper operation. The circuit of Fig. 3f serves as an impedance multiplier. That of Fig. $3 g$ behaves as a super inductor and that of Fig. 3h as a FDNR.

\section{First-Order Filter Structures}

A voltage variable integrator structure with a differential input is shown in Fig. 4a. The integrator serves as the basic building block in many filter structures. Two different lossy integrators (first-order lowpass filters) are shown in Figs. $4 \mathrm{~b}$ and $4 \mathrm{c}$. The 


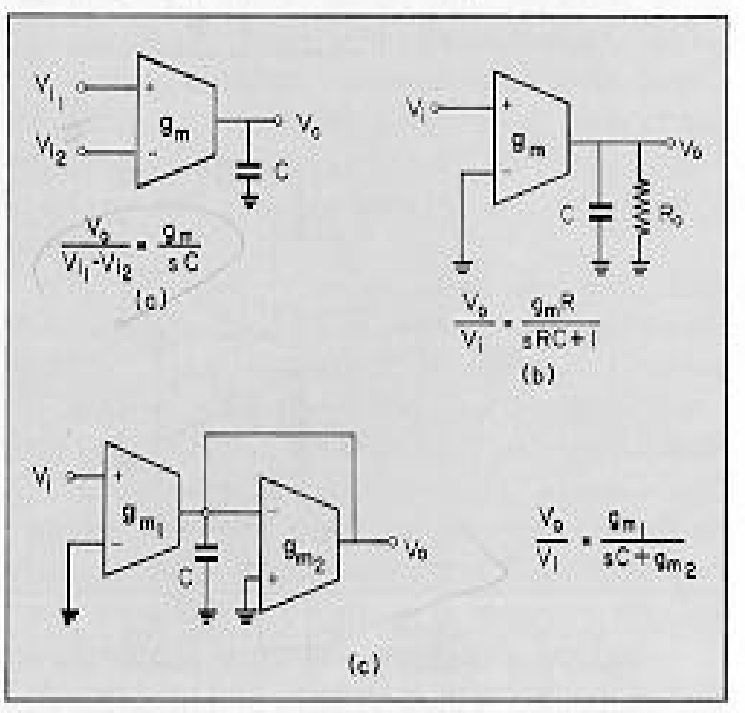

Figure 4. Integrator structures. (a) Simple. (b) Lossy. (c) Adjustable.

circuit of Fig. $4 \mathrm{~b}$ has a loss that is fixed by the $R C$ product and a gain controllable by $g_{m}$. The circuit of Fig. $4 \mathrm{c}$ offers considerably more flexibility. The pole frequency can be adjusted by $g_{m 2}$ (interchanging the input terminals of OTA 2 actually allows the pole to enter the right half plane), and the dc gain can be subsequently adjusted by $g_{m}$. It should be noted that the structure of Fig. $4 \mathrm{c}$ contains no resistors and can be obtained from the circuit of Fig. $4 \mathrm{~b}$ by replacing the resistor $R$ with the controlled impedance of Fig. 3a. Another lossy integrator without adjustable gain but with adjustable pole location and a very simple structure is shown in Fig. 5a.

When designing cascaded integrator-based filter structures, it may be the case that the input impedance to some stages is not infinite. If that be the case, a unity gain buffer would be required for coupling, since the output impedances of all integrators in Fig. 4 are nonzero. Note, however, that no buffer is needed for the cascade of any of the integrators of Fig. 4, since the input impedance to each circuit is ideally infinite.

First-order filters can be readily built using OTAS. Considerable flexibility in controlling those specific filter characteristics that are usually of interest is possible with these structures. Several first-order voltage-controlled filters are shown in Fig. 5, and a functional plot of the transfer characteristics as a function of the transconductance gains is shown in Fig. 6.
The $3 \mathrm{~dB}$ cutoff frequency of the lowpass filter of Fig. 5a is given by the expression

$$
\mathrm{f}_{3 \mathrm{~dB}}=\frac{\mathrm{g}_{\mathrm{m}}}{2 \pi \mathrm{C}}
$$

Linear adjustment of $f_{\mathrm{dB}}$ with $\mathrm{g}_{\mathrm{m}}$ is attainable with this circuit while maintaining a unity de frequency gain. The structure of Fig. 5b has a fixed pole location and adjustable $\mathrm{dc}$ gain with the transconductance gain $\mathrm{g}_{\mathrm{n}}$. If the resistor in this circuit is replaced with the controlled resistor of Fig. $3 \mathrm{a}$, the circuit would have independently adjustable gain and break frequency. The highpass structure of Fig. $5 \mathrm{c}$ also has a $3 \mathrm{~dB}$ cutoff frequency given by

$$
\mathrm{f}_{3 \mathrm{~dB}}=\frac{\mathrm{g}_{\mathrm{m}}}{2 \pi \mathrm{C}}
$$

It can be observed that the characteristic networks for the lowpass and highpass structures of Figs. $5 \mathrm{a}$ and $5 \mathrm{c}$ are identical, and thus they have the same pole structures. They differ only in where the excitation is applied.

The circuits of Figs. $5 \mathrm{~d}$ and $5 \mathrm{e}$ act as shelving equalizers. The response of both circuits can be continuously changed from lowpass to allpass to highpass by adjusting $\mathrm{g}_{\mathrm{m}}$ as can be seen from Fig. 6 . The basic difference in the two circuits is that the former has a fixed pole and adjustable zero, whereas the circuit of Fig. 5e has an adjustable pole and fixed zero. As for the circuit of Fig. 5b, additional flexibility can be obtained if the grounded resistor in the circuit of Fig. $5 \mathrm{~d}$ is replaced with the controlled resistor of Fig. $3 \mathrm{a}$.

The circuit of Fig. $5 \mathrm{f}$ acts as a lowpass filter with highfrequency gain determined by the $C_{1}: C_{2}$ ratio. Both the pole and zero in this circuit are adjustable through the parameter $g_{m}$ but the ratio is held constant. This preserves the shape in the transfer characteristics and thus represents only a frequency shift in the response, as shown in Fig. 6 f.

The circuit of Fig. 5g utilizes an additional OTA and offers considerable flexibility. If either $g_{m 1}$ or $g_{m_{2}}$ fixed, the circuit behaves much like the shelving equalizers discussed above. If $g_{m 1}$ and $g_{m 2}$ are adjusted simultaneously, then a fixed pole-zero ratio and, hence, shape preserving response is possible. In this case, the circuit can be lowpass, allpass, or highpass, depending upon the $g_{m 1}: g_{m 2}$ ratio. If the "+" and "." terminals of $g_{m 1}$ are interchanged and the transconductance gains are adjusted so that $g_{m 1}=g_{m 2}$, the circuit behaves as a phase equalizer. 


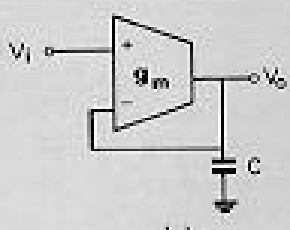

(a)
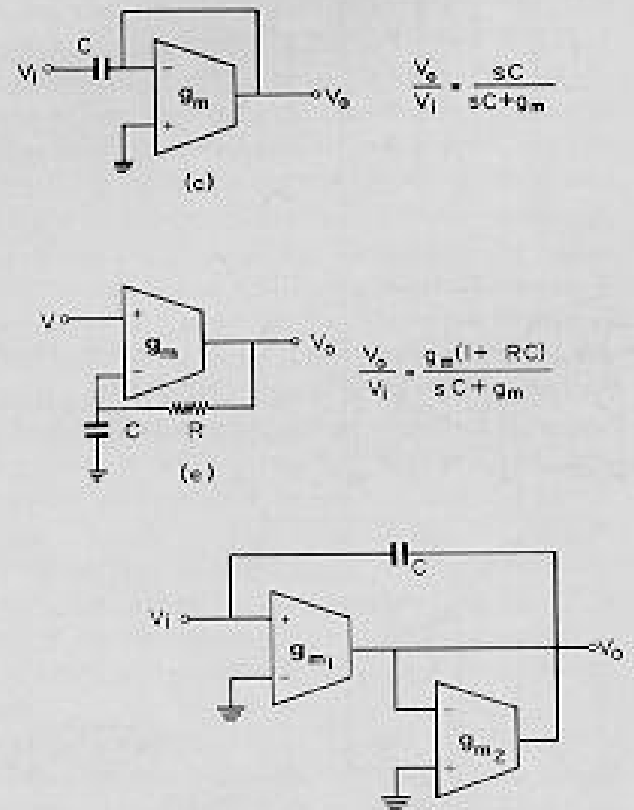

(g)
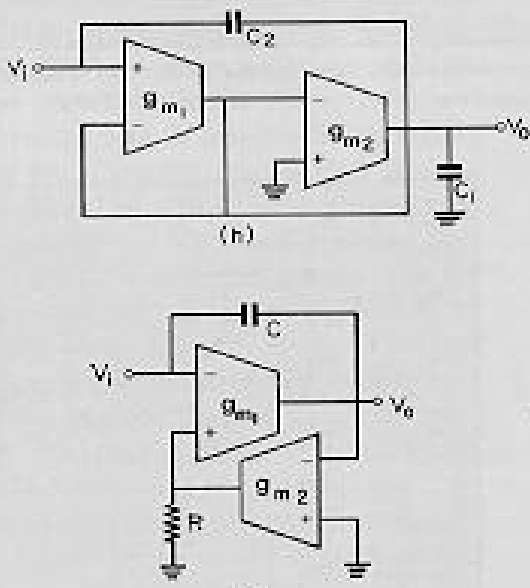

(1)

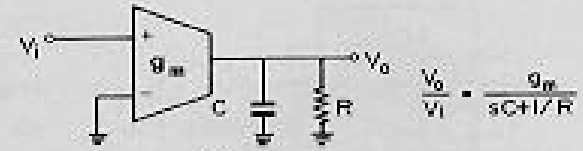

(b)

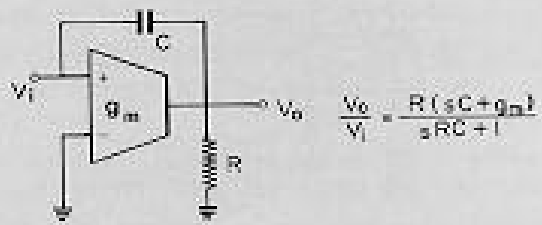

(d)

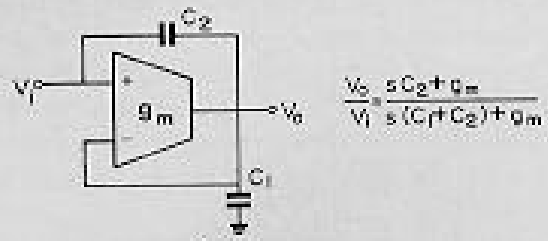

(1)
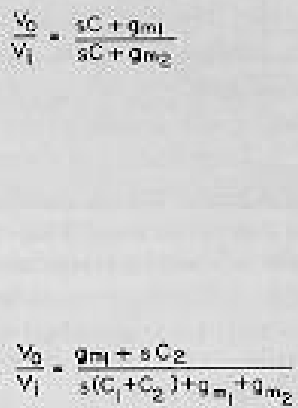

$\frac{V_{0}}{V_{i}} \cdot \frac{s C-g n_{i}}{s C+9 m_{1} 9 m_{2} R}$

$\theta_{m} R=1$

Fig. 5 First-order voltage-controlled filters. (a) Lowpass, fixed dc gain pole adjustable. (b) Lowpass fixed pole, adjustable dc gain. (c) Highpass, fixed high-frequency gain, adjustable pole. (d) Shelving equalizer, fixed high-frequency gain, fixed pole, adjustable zero. (e) Shelving equalizer, fixed high-frequency gain, fixed zero, adjustable pole. (f) Lowpass filter adjustable pole and zero, fixed ration. (g) Shelving equalizer, independently adjustable pole and zero. (h) Lowpass or highpass filter, adjustable zero and pole, fixed ratio or independent adjustment. (i) Phase shifter, adjustable with $g_{m}$. 
The circuit of Fig. 5h also preserves the shape of the transfer function, provided $g_{m 1}$ and $g_{m 2}$ are adjusted in such a manner that their ratio remains constant. In this case, the shape of the response is determined by the $g_{m 1}: g_{m 2}$ and $C_{1}: C_{2}$ ratio. Depending upon these ratios, the response is either lowpass or highpass in nature, as indicated in Fig. $6 \mathrm{~h}$.

If $g_{m 2} \mathrm{R}=1$, the circuit of Fig. $5 \mathrm{i}$ behaves as a phase equalizer, $g_{m 1}$ can be used to adjust the phase shift. For monolithic applications, the resistor $R$ can be replaced with a third OTA, using the configuration of Fig. 3a.

\section{Second-Order Structures}

Second-order filter structures find widespread applications directly and in the design of higher-order filters. Although the emergence of practical voltage or current-controlled first-order filters and amplifiers has been slow, even fewer techniques exist for the design of controlled second- and higher-order structures. Switchedcapacitor techniques have been successfully used to build voltage-controlled filter structures by building a voltagecontrolled oscillator and using the output as the required clock for the switching of the capacitors. Although useful in some applications, these structures are not continuous time in nature, have limited dynamic range, and are limited to reasonably low-frequency applications. Concentration here will be on continuous-time voltage controlled structures.

One common requirement in the design of voltage controlled filter structures is that the filter characteristics be adjusted in a manner that essentially results in frequency scaling. In all-pole applications, such as the lowpass Butterworth and Chebyschev case, as well as the bandpass and highpass versions of these approximations, the frequency scaling is tantamount to moving all poles a prescribed distance in a constant- $Q$ manner. Those familiar with active filter structures will recall that pole movement in second-order structures through the adjustment of a single component is always on a circular path (constant $\omega_{0}$ ) or on a straight line (constant bandwidth) parallel to the imaginary axis in the splane. The challenges associated with constant- $Q$ pole adjustment through the simultaneous tuning of two or more components should be obvious.

A seemingly more difficult situation exists when considering the design of the popular elliptic filters. To maintain the elliptic characteristics as the cutoff frequency is changed, all poles and all zeros of the approximating function must be moved simultaneously and with the appropriate ratio in a constant- $Q$ manner.

A group of second-order voltage-controlled filter structures are discussed in this section. Circuits with constant- $Q$ pole adjustment, circuits with constant bandwidth $\omega_{0}$, adjustment, and circuits with independent pole and zero adjustment are presented. Some circuits with simultaneous constant- $Q$ adjustment of both the poles and zeros are also presented along with a general biquadratic structure. These structures have immediate applications in voltage-controlled Butterworth, Chebyschev, and Elliptic designs.

A simple second-order filter structure is shown in Fig. 7a [17], [19]. This structure is canonical in the sense that only four components are needed to obtain second-order transfer functions. The output voltage, $V_{o}$, is given by the expression

$$
V_{\mathrm{ol}}=\frac{\mathrm{s}^{2} \mathrm{C}_{1} \mathrm{C}_{2} \mathrm{~V}_{\mathrm{C}}+\mathrm{sC}_{1} \mathrm{~g}_{\mathrm{m} 2} \mathrm{~V}_{\mathrm{B}}+\mathrm{g}_{\mathrm{m} 1} \mathrm{~g}_{\mathrm{m} 2} \mathrm{~V}_{\mathrm{A}}}{\mathrm{s}^{2} \mathrm{C}_{1} \mathrm{C}_{2}+\mathrm{sC}_{1} \mathrm{~g}_{\mathrm{m} 2}+\mathrm{g}_{\mathrm{ml}} \mathrm{g}_{\mathrm{m} 2}}
$$

The transfer function for the specific excitations at $V_{A}, V_{B}$, and $\mathrm{V}_{C}$ are listed in the Table. Note that for $g_{m 1}=g_{m 2}=g_{m}$, the lowpass, bandpass, highpass, and notch versions of this circuit all behave as $\omega_{o}$ adjustable circuits with fixed pole $Q^{\prime}$ s. The pole $Q^{\prime}$ s are determined by the capacitor ratio, which can be accurately maintained in monolithic designs. It is interesting to note that the zeros of the notch circuit also move in a constant- $Q$ (i.e. along the $j w$ axis) manner with the poles, as $g_{m}$ is adjusted.

Occasionally, it is desirable to have circuits in which $\omega_{0}$ and $Q$ of the poles can be independently adjusted. Two circuits with these characteristics are shown in Fig. 7b [18], [19], [24] and Fig. 7c [18], [24]. The output voltages for these circuits are, respectively,

$$
V_{\mathrm{o} 2}=\frac{\mathrm{s}^{2} \mathrm{C}_{1} \mathrm{C}_{2} \mathrm{~V}_{\mathrm{C}}+\mathrm{sC}_{1} \mathrm{~g}_{\mathrm{m} 2} \mathrm{~V}_{\mathrm{B}}+\mathrm{g}_{\mathrm{m} 1} \mathrm{~g}_{\mathrm{m} 2} \mathrm{~V}_{\mathrm{A}}}{\mathrm{s}^{2} \mathrm{C}_{1} \mathrm{C}_{2}+\mathrm{sC}_{1} \mathrm{~g}_{\mathrm{m} 2} \mathrm{~g}_{\mathrm{m} 3} \mathrm{R}+\mathrm{g}_{\mathrm{m} 1} \mathrm{~g}_{\mathrm{m} 2}}
$$

and

$$
V_{\mathrm{o} 3}=\frac{\mathrm{s}^{2} \mathrm{C}_{1} \mathrm{C}_{2} \mathrm{~V}_{\mathrm{C}}+\mathrm{sC}_{1} \mathrm{~g}_{\mathrm{m} 2} \mathrm{~V}_{\mathrm{B}}+\mathrm{g}_{\mathrm{m} 1} \mathrm{~g}_{\mathrm{m} 2} \mathrm{~V}_{\mathrm{A}}}{\mathrm{s}^{2} \mathrm{C}_{1} \mathrm{C}_{2}+\mathrm{sg}_{\mathrm{m} 3} \mathrm{C}_{1}+\mathrm{g}_{\mathrm{m} 1} \mathrm{~g}_{\mathrm{m} 2}}
$$




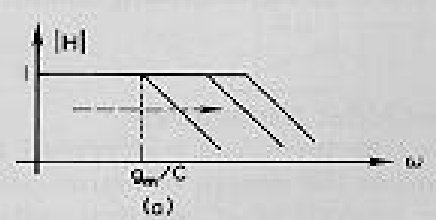

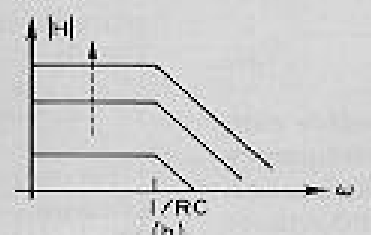

(b)

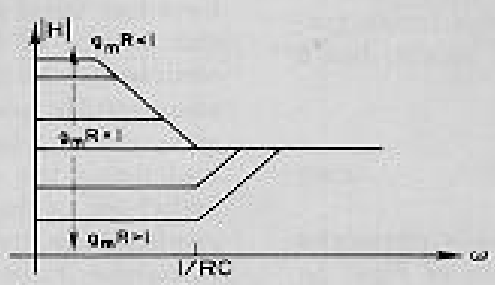

(e)

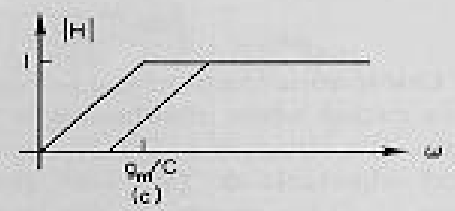

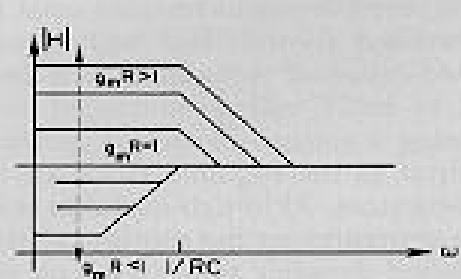

(d)

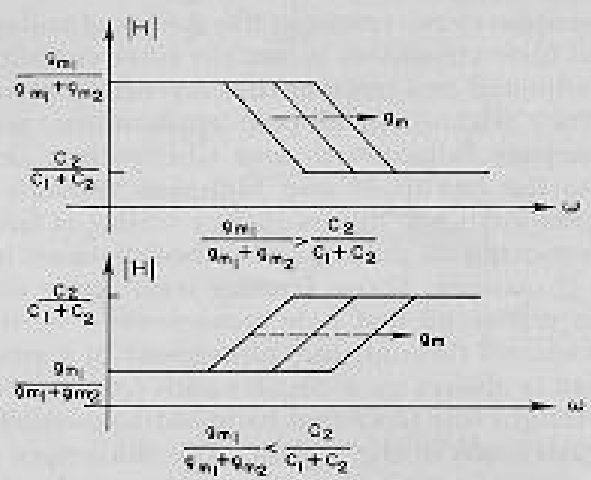

(h)

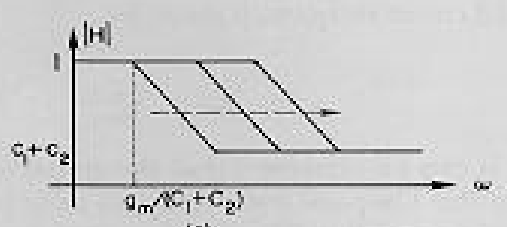

(f)

Fig. 6 Transfer characteristics for first-order structures of Fig. 5. (a) Circuit of Fig. 5a. (b) Circuit of Fig. 5b. (c) Circuit of Fig. 5c. (d) Circuit of Fig. 5d. (e) Circuit of Fig. 5e. (f) Circuit of Fig. 5f. (g) Circuit of Fig. 5g. (h) Circuit of Fig. 5h. (i) Circuit of Fig. $5 i$.

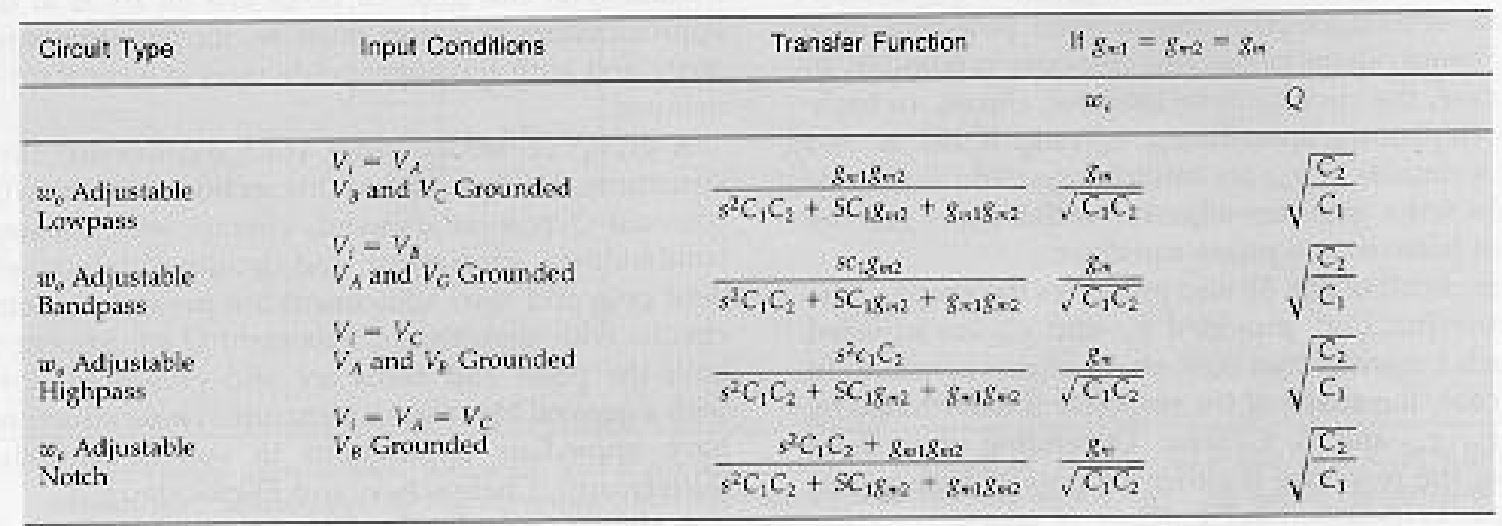

Table Transfer functions for biquadratic structure of Fig. $7 a$. 

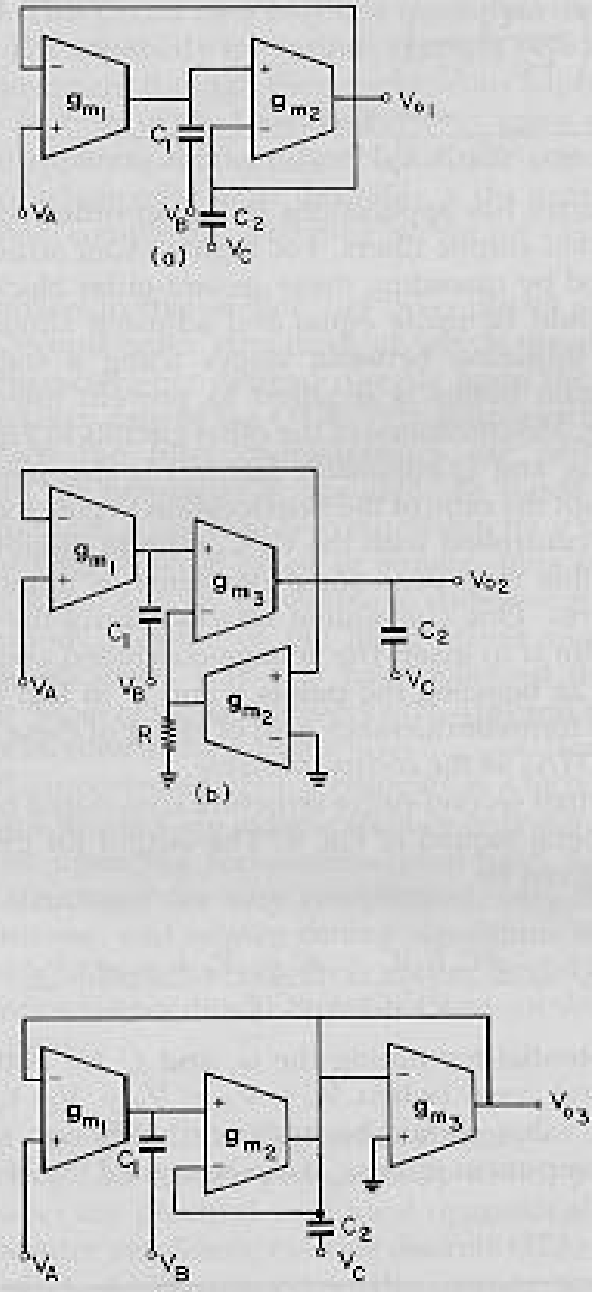

(c)

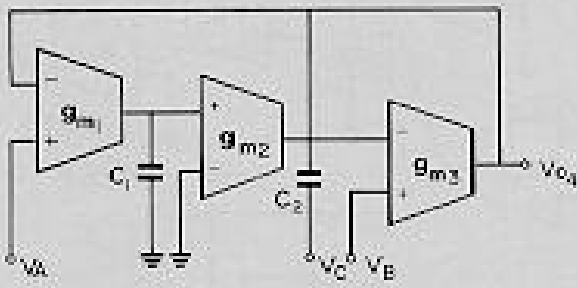

(d)

Fig. 7 Second-order filter structures.
The circuits of Figs. $7 \mathrm{~b}$ and $7 \mathrm{c}$ can be also used to implement lowpass, bandpass, highpass, and notch transfer functions through the proper selection of the inputs as for the circuit of Fig. $7 \mathrm{a}$.

In the circuit of Fig. 7b, the expressions for $\omega_{0}$ and $Q$ of the poles of the circuit are given by

$$
\omega_{\mathrm{o}}=\sqrt{\frac{\mathrm{g}_{\mathrm{m} 1} \mathrm{~g}_{\mathrm{m} 2}}{\mathrm{C}_{1} \mathrm{C}_{2}}}
$$

and

$$
\mathrm{Q}=\frac{1}{\mathrm{~g}_{\mathrm{m} 3} \mathrm{R}} \sqrt{\frac{\mathrm{C}_{2} \mathrm{~g}_{\mathrm{m} 1}}{\mathrm{C}_{1} \mathrm{~g}_{\mathrm{m} 2}}}
$$

The poles can be moved in a constant-Q manner if $g_{m 3}$ is fixed, and if $g_{m 1}=g_{m 2}=g_{m}$ is adjusted; whereas movement in a constant $\omega_{0}$ manner is attainable if $g_{m 3}$ is adjusted when $g_{m 1}$ and $g_{m 2}$ remain constant. The independent adjustment of $\omega_{0}$, and $Q$ is apparent.

For the circuit of Fig. 7c, the expressions for $\omega_{0}$ and $Q$ of the poles become

$$
\begin{gathered}
\omega_{\mathrm{o}}=\sqrt{\frac{\mathrm{g}_{\mathrm{m} 1} \mathrm{~g}_{\mathrm{m} 2}}{\mathrm{C}_{1} \mathrm{C}_{2}}} \\
Q=\left(\sqrt{\frac{\mathrm{C}_{2}}{\mathrm{C}_{1}}}\right) \frac{\sqrt{\mathrm{g}_{\mathrm{m} 1} \mathrm{~g}_{\mathrm{m} 2}}}{\mathrm{~g}_{\mathrm{m} 3}}
\end{gathered}
$$

$\omega_{0}$ can be adjusted linearly with $g_{m 1}=g_{m 2}=g_{m}$ and $g_{m 3}$ constant. Such movement is often termed constant bandwidth movement. If $g_{m 1}, g_{m 2}$, and $g_{m 3}$ are adjusted simultaneously, constant- $Q$ pole movement is possible. Adjusting $g_{m 3}$ (for $\mathrm{Q}>1 / 2$ ) moves the poles along vertical lines parallel to the $j w$ axis in the s-plane.

The circuit of Fig. $7 \mathrm{~d}$ has an output given by

$$
V_{\mathrm{o} 4}=\frac{\mathrm{V}_{\mathrm{C}} \mathrm{C}_{1} \mathrm{C}_{2} \mathrm{~s}^{2}+\mathrm{V}_{\mathrm{B}} \mathrm{g}_{\mathrm{m} 3} \mathrm{sC}_{1}+\mathrm{g}_{\mathrm{m} 1} \mathrm{~g}_{\mathrm{m} 2} \mathrm{~V}_{\mathrm{A}}}{\mathrm{s}^{2} \mathrm{C}_{1} \mathrm{C}_{2}+\mathrm{sC}_{1} \mathrm{~g}_{\mathrm{m} 3}+\mathrm{g}_{\mathrm{m} 1} \mathrm{~g}_{\mathrm{m} 2}}
$$

The $\omega_{\mathrm{o}}$ and $Q$ of the poles are, respectively, 


$$
\begin{gathered}
\omega_{\mathrm{o}}=\sqrt{\frac{\mathrm{g}_{\mathrm{m} 1} \mathrm{~g}_{\mathrm{m} 2}}{\mathrm{C}_{1} C_{2}}} \\
Q=\frac{1}{\mathrm{~g}_{\mathrm{m} 3}} \sqrt{\frac{\mathrm{g}_{\mathrm{m} 1} \mathrm{~g}_{\mathrm{m} 2} C_{2}}{\mathrm{C}_{1}}}
\end{gathered}
$$

Although the transfer function is similar to that above, note that since the coefficient of the s term in the numerator equals that in the denominator, adjustment of the bandpass version of this circuit with $g_{m 1}=g_{m 2}=g_{m}$ will result in a constant bandwidth, constant gain response.

For monolithic structures, it may prove useful to replace the resistor in Fig. 7b with the OTA structure of Fig. 3a. Likewise, if the bandwidth adjustment with $g_{m 3}$ is not needed, it may be desirable to replace the third OTA shown in Fig. 7c with a fixed resistor in some applications.

Phase equalizers are also possible with the structures shown in Fig. 7. For example, interchanging the " + " and " - " terminals of the first two OTAs in Fig. 7c, setting $V_{\mathrm{A}}=$ $V_{B}=V_{C}=V_{i}$, and making $g_{m 1}=g_{m 2}=g_{m 3}=g_{m}$ results in a second-order $g_{m}$ adjustable phase equalizer.

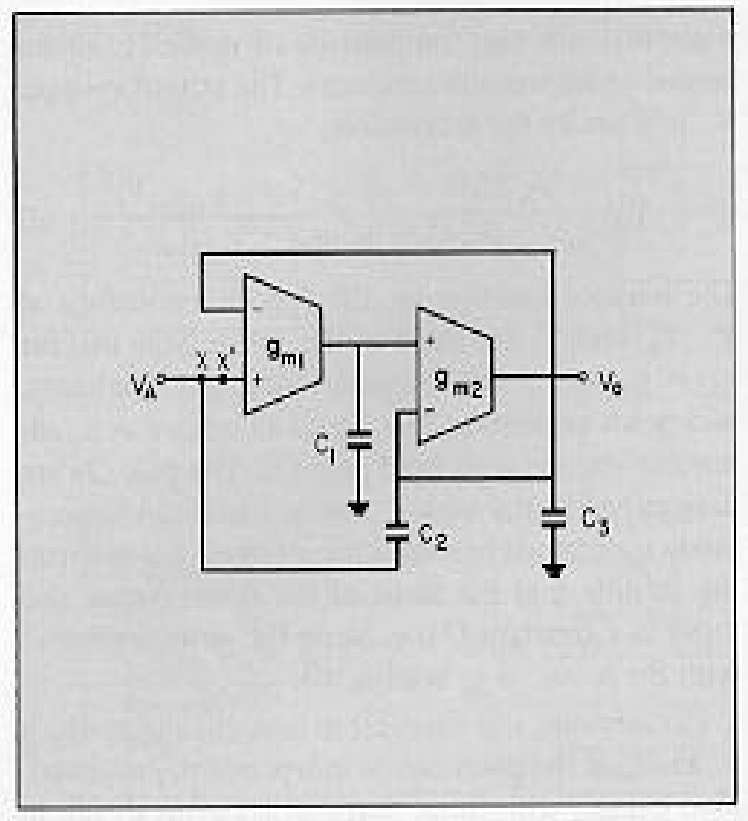

Fig. 8 Elliptic Filter structure.

The circuit of Fig. 8 has both poles and zeros that can be adjusted simultaneously in a constant $Q$ manner. The circuit is similar to those shown in Fig. 7a with the exception that the capacitor $C_{2}$ in the previous circuits has been split to allow for adjusting the pole-zero ratio. The transfer function of the circuit is given by

$$
\begin{aligned}
& \frac{V_{o}}{V_{i}}=\left(\frac{C_{2}}{C_{2}+C_{3}}\right) \\
& \left(\frac{s^{2}+g_{m 1} / C_{1} C_{2}}{s^{2}+s_{m} /\left(C_{2}+C_{3}\right)+g_{m 1} g_{m 2} / C_{1}\left(C_{2}+C_{3}\right)}\right)
\end{aligned}
$$

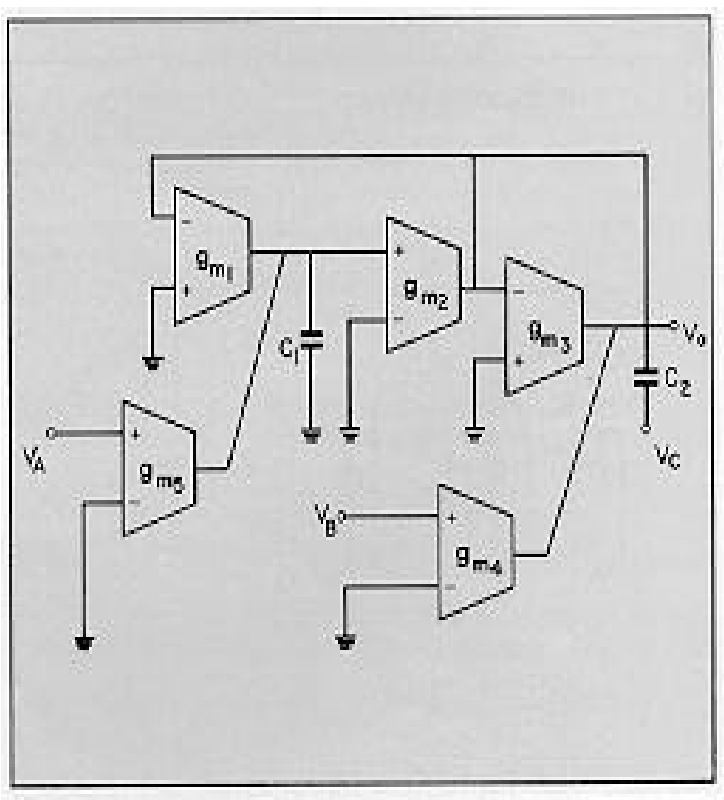

Fig. 9 General biquadratic structure.

This circuit has applications in higher-order voltagecontrolled elliptic filters. For higher-order structures obtained by cascading these second-order blocks, all $g_{m}$ 's would be made equal and adjusted simultaneously. Buffering between stages using a standard unity gain buffer is required to prevent interstage loading. Modifications of the other circuits in Fig. 7 to obtain $\omega_{0}$ and $Q$ adjustable features is also possible. Although the ratio of the zero location to pole location can be controlled with the $C_{2} / C_{3}$ ratio in discrete designs, this may pose some problems in monolithic structures. One convenient way to control the pole-zero ratio is to insert the voltage-controlled amplifier of Fig. $2 \mathrm{~g}$ between the points $\mathrm{x}$ and $\mathrm{x}$ ' in Fig. 8 and use the transconductance gain of either of these additional OTAs as the control variable.

The final second-order structure considered here is the general biquad of Fig. 9. The output for this circuit is given by

$\mathrm{V}_{\mathrm{o}}=\frac{\mathrm{s}^{2} \mathrm{C}_{1} \mathrm{C}_{2} \mathrm{~V}_{\mathrm{C}}+\mathrm{sC}_{1} \mathrm{~g}_{\mathrm{m} 4} \mathrm{~V}_{\mathrm{B}}+\mathrm{g}_{\mathrm{m} 2} \mathrm{~g}_{\mathrm{m} 5} \mathrm{~V}_{\mathrm{A}}}{\mathrm{s}^{2} \mathrm{C}_{1} \mathrm{C}_{2}+\mathrm{sC}_{1} \mathrm{~g}_{\mathrm{m} 3}+\mathrm{g}_{\mathrm{m} 2} \mathrm{~g}_{\mathrm{m} 1}}$ 


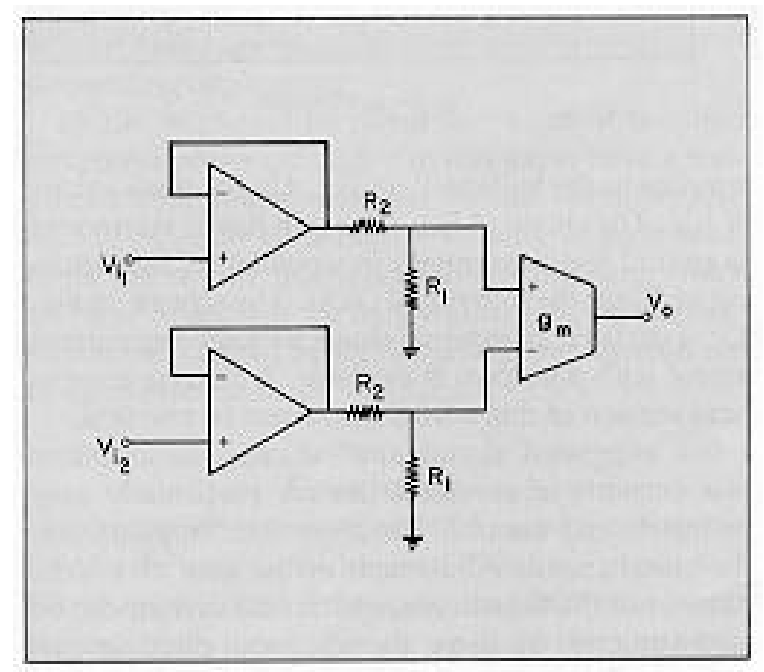

Fig. 10 Signal conditioner for OTAs.

The potential for tuning the w. and $Q$ for both the poles and zeros (when $V_{i}=V_{A}=V_{B}=V_{C}$ ) to any desired value should be apparent. Although somewhat component intense, it can be argued that if there is to be capability for completely arbitrary location of a pair of poles and a pair of zeros via adjustment of the transconductance gain of the OTA, then at least 4 degrees of freedom and, hence, 4 OTAs are required. This circuit uses only one more than the minimum! The capability for various types of pole and/or zero movement through the simultaneous adjustment of two or more of the transconductance gains should also be apparent. Many other biquadratic structures, some of which offer more flexibility at the expense of additional complexity, also exist but are not discussed here.

Emphasis in this section has been placed entirely upon second-order structures in which the desired filter characteristics depend directly upon the transconductance gain of the OTA. Very simple structures in which the filter characteristics are adjustable through the parameter $g_{m}$ resulted. As stated in the introduction, $g_{m}$ is readily controllable by a dc bias current over a wide range of values, thus making these circuits directly applicable to voltagecontrolled applications. Several of the more recent works on OTA applications [18]-[24] have followed this approach. Most of the earlier works [7]-[16] and the circuits presented in the manufacturer's application notes [3]-[5] concentrated upon topologies in which the filter characteristics are independent or only mildly dependent upon the transconductance gain. Most of these structures are very complicated, very component-intense, and require tuning algorithms that are unwieldy. Alternatives to these earlier designs using conventional operational amplifiers have proven to be much better.

\section{Practical Considerations}

Although all circuits presented up to this point in this paper are practical with ideal operational transconductance amplifiers, existing discrete OTAs are far from ideal. As mentioned in the introduction, the major limiting factor with commercially available OTAs is the limited differential input voltage swing. Recent activity in the literature has concentrated upon designing OTAs with improved input characteristics [27]-[28]. Significant improvements in performance over what is currently available with discrete OTAs have been demonstrated. An alternative is to use voltage attenuators and buffers at the input of existing OTAS. This technique is often suggested in the manufacturer's application notes and is illustrated in Fig. 10. This technique can be used to obtain reasonable signal swings with all circuits discussed up to this point. Although such circuits are useful, a rather high price is paid for this modification. First, the circuit requires many more components. Second, the finite bandwidth of the op amps will limit the frequency response of the OTA structures. Finally, the attenuation of the input signal to the OTA causes

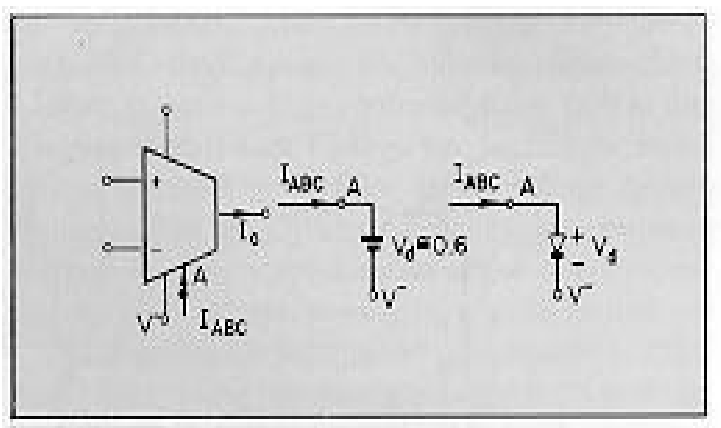

Fig. 11 Macromodel of bias current port on bipolar OTA.

a serious loss in dynamic range. From a topological point of view, some OTA based structures are inherently more susceptible to differential voltage limitations than others. This parallels the concern for op amp based active $R C$ and switched-capacitor 

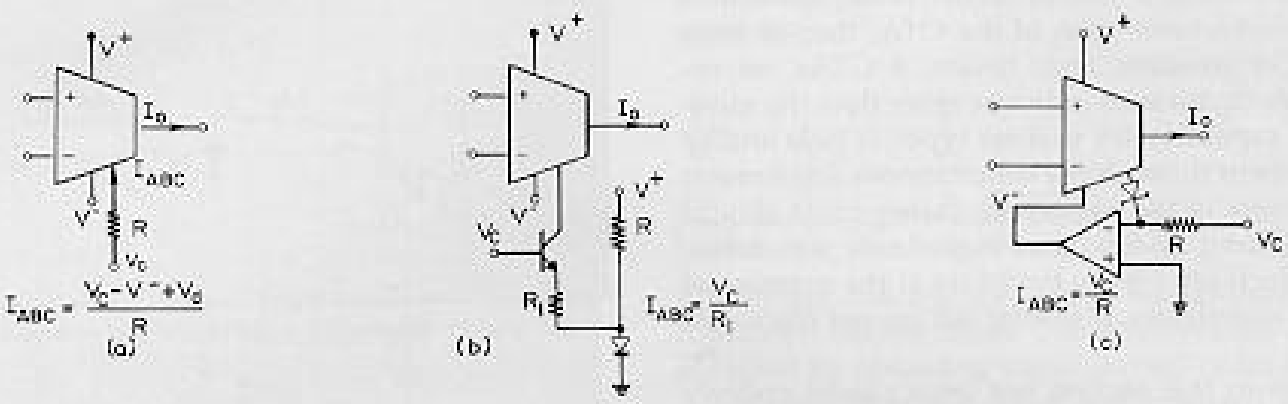

(c)

Fig. 12 Schemes for obtaining voltage control with the OTA.

thus, the $g_{m}$ of the OTA by an external control voltage, $V_{c}$,

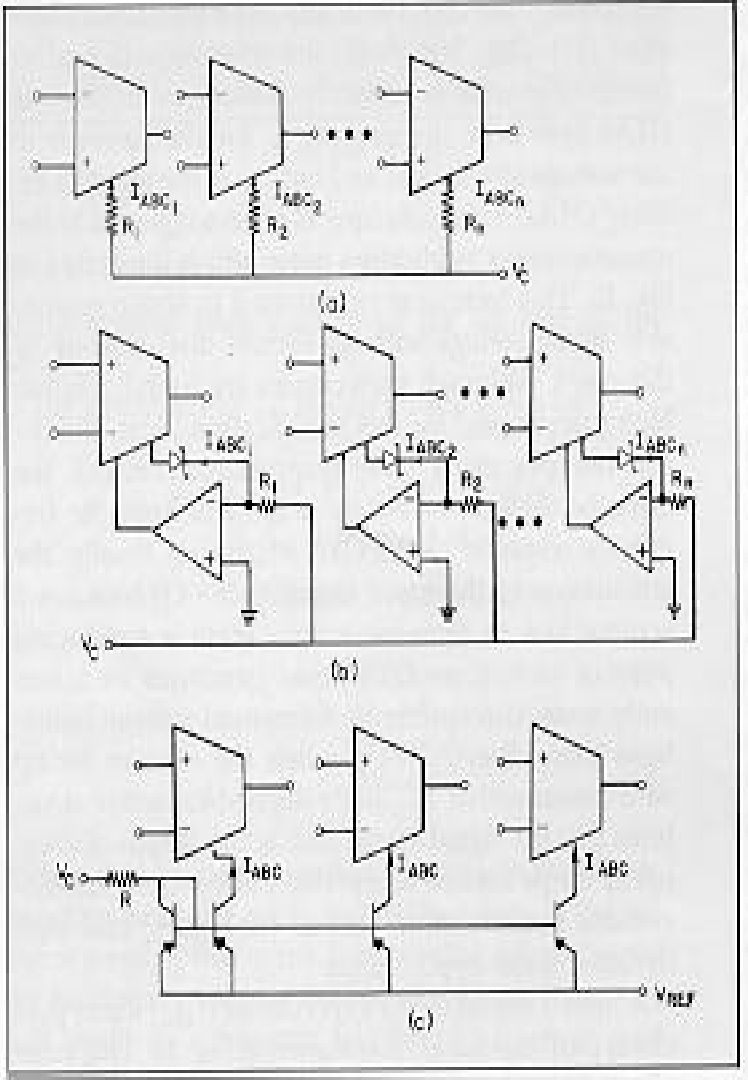

Fig. 13 Schemes for simultaneous $g_{m}$ adjustment.

structures that the signal amplitudes at the output of internal op amps assume acceptable values. These considerations become more serious for high $Q$ and high dynamic range applications.

A macro model of the bias current $\left(I_{\mathrm{ABC}}\right)$ input port of a typical bipolar OTA is shown in Fig. 11. This actually forms part of an internal current mirror that is discussed later. Several schemes for controlling the current $\left(I_{\mathrm{ABC}}\right)$ and,

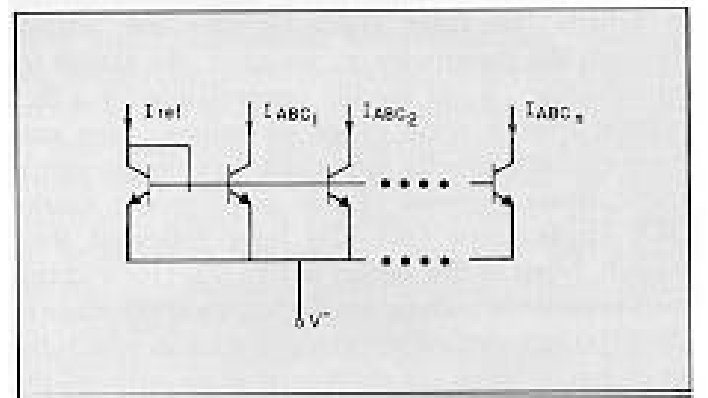

are shown in Fig. 12. The

Fig. 14 Single input-multiple output bias current

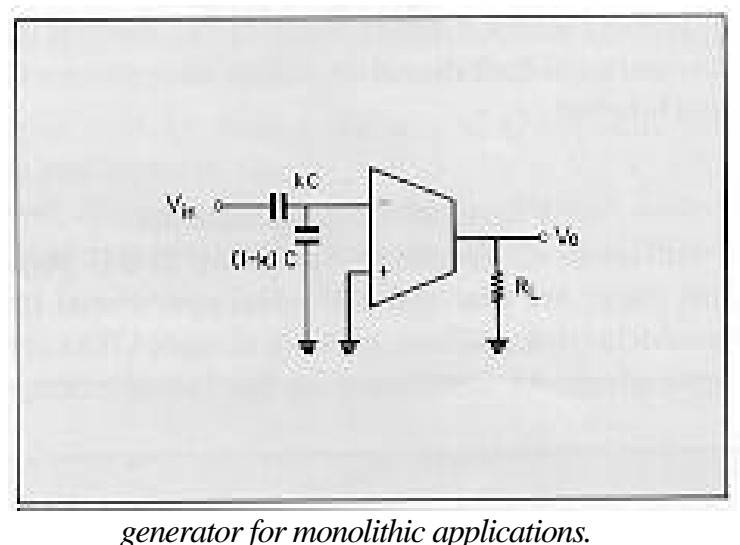

Fig. $15 g_{m}$ attenuator.

first circuit is the simplest but is very sensitive to small changes of $V_{c}$ as $V_{c}$ approaches $.6 v+V$. In the second circuit, the control voltage is referenced to zero but the small $V_{c}$ is sensitive to mismatches between the B-E voltage of the transistor and the forward diode voltage drop. In the circuit of Fig. 12c, the control voltage is also referenced to ground and is not dependent upon the matching or cancellation of voltages across external forward biased $p n$ 
junctions. The zener diode is used to maintain the common mode voltage at a reasonable level. The frequency response of the op amp is not of concern here since it is used only in the dc control path. It should be noted that the amplifier bias current is proportional to $V_{c}$ for all schemes shown in Fig. 12. Since $I_{\mathrm{ABC}}$ can typically be adjusted over several decades, all schemes will be very sensitive to small changes in $V_{c}$ toward the low current end of the $I_{\mathrm{ABC}}$ range. Logarithmic amplifiers are often used to control $I_{\mathrm{ABC}}$ with an external control voltage if the wide adjustment range of $I_{\mathrm{ABC}}$ is to be effectively utilized.

Many of the filter circuits discussed in the previous sections of this paper require the simultaneous adjustment of matched $g_{m}$ 's. Several schemes for achieving this are shown in Fig. 13. In the first circuit, it is easy to adjust the $g_{m}$ 's by trimming the resistors for a fixed $g_{m}$. The circuit is quite sensitive to the slight differences in the voltage $V_{d}$ of Fig. 11a for small values of $I_{\mathrm{ABC}}$. The circuit of Fig. 13b again has $V_{c}$ referenced to ground and is essentially independent of the matching of $V_{d}$ for the individual OTAs. The scheme of Fig. 13c is useful if an external single package pnp current mirror with $n$ outputs is available. A discrete component version of this mirror would not be practical.

For integrated circuit applications, the amplifier bias currents of several OTAs are particularly easy to match and control. For monolithic applications, the simultaneous adjustment of the gain of a large number of OTAs with a single dc bias current can be easily attained by using a single input-multiple output current mirror such as is shown in Fig. 14. This structure actually replaces the bias current mirrors on each of the OTAS. The transconductance gains can be ratioed, if desired, by correspondingly ratioing the emitter areas (or width length ratio for MOS structures) in the outputs of the current mirror.

With conventional operational amplifiers, the slew rate, input impedance, output impedance, and maximum output current are essentially fixed at the design stage. For OTAS, it is generally the case that these parameters are either proportional or inversely proportional to $I_{\mathrm{ABC}}$. Thus adjusting $g_{m}$ via $I_{\mathrm{ABC}}$ causes all of these parasitic parameters to change accordingly. Although the user should be cognizant of the changes in these parameters, the problems they present are manageable. The output capacitance of an OTA does cause concern at low output currents and high frequencies.

Much as in the design of conventional op amp based circuits, the designer must allow for a dc bias current path for both input terminals of the OTA. Although the amplifier of Fig. 15 serves as an effective $g_{m}$ attenuator, which will prove useful in some applications, the circuit is useless since the required input bias current will cause an accumulation of charge on the capacitors and eventual saturation of the OTA. The reader should be cautioned that more complicated circuits with the same problem are suggested in the literature [17].

Numerous nonlinear applications of OTA structures exist. Suffice it to say that since the amplifier bias current, $I_{\mathrm{ABC}}$ can be considered as a third signal input, simple multipliers, modulators, and a host of other nonlinear circuits are possible. The reader is referred to the application notes for a discussion of some of the nonlinear applications. Some of the structures that use only OTAs and capacitors show promise for monolithic applications in MOS or bipolar processes. The circuits should offer highfrequency continuous-time capabilities. Either external voltage-control or an internal reference circuit to compensate for process and temperature variations will be necessary to make these circuits practical in demanding applications.

Finally, it should be noted that some of the filter structures presented earlier in this paper have a non-infinite input impedance, and that the output impedance is generally quite high. Cascading of such structures will require interstage buffer amplifiers, which will tend to degrade the bandwidth of the overall filter structures. Output buffers are also generally required to drive external loads.

\section{Conclusions}

A group of voltage-controlled circuits using the OTA as the basic active element have been presented. The characteristics of these circuits are adjusted with the externally accessible dc amplifier bias current. Most of these circuits utilize a very small number of components. Applications include amplifiers, controlled impedances, and filters. Higher-order continuous-time voltage-controlled filters such as the common Butterworth, Chebyschev, and Elliptic types can be obtained. In addition to the voltagecontrol characteristics, the OTA based circuits show promise for high-frequency applications where conventional op amp based circuits become bandwidth limited.

The major factor limiting the performance of OTA based filters using commercially available OTAs is the severely limited differential input voltage capability inherent with conventional differential amplifier input stages. Recent research results suggested significant improvements in the input characteristics of OTAs can be attained [27]-[28].

\section{References}


[1] H. A. Wittlinger, "Applications of the CA3080 and CA3080A High Performance Operational Transconductance Amplifiers," RCA Application Note ICAN-6668.

[2] C. F. Wheatley and H. A. Wittlinger, "OTA Obsoletes OP. AMP," P. Nat. Econ. Conf. pp. 152-157, Dec. 1969.

[3] RCA Electronic Components, Linear Integrated Circuits, Model CA3060: Data File 404, Mar. 1970.

[4] National Semiconductor, Linear Applications Handbook, 1980.

[5] RCA Solid-State Division, Data Book, Linear Integrated Circuits, File No. 475, Mar. 1975.

[6] M. Bialko and R. W. Newcomb, "Generation of All Finite Linear Circuits Using the Integrated DVCCS," IEEE Trans. on Circuit Theory, vol. CT-18, pp. 733 736, Nov. 1971.

[7] M. Bialko, W. Sienko, and R. W. Newcomb, "Active Synthesis Using the DVCCS/DVCVS," Int. J. of Circuits Theory App., vol. 2, pp. 23-28, 1974.

[8] S. Franco, "Use Transconductance Amplifier to Make Pro-

grammable Active Filters," Electronic Design, vol. 24, pp. 98-101, Sept. 1976.

[9] F. Atiya, A. Soliman, and T. Saadawi, "Active RC Bandpass and Lowpass Filters Using the DVCCS/DVCVS," Electron. Lett. vol. 12, pp. 360-361, July 1976.

[10] F. Anday, "On the Analysis and Synthesis of Active Networks Containing DVCCS/DVCVS," Proc. IEEE, pp. 375-376, Mar. 1976.

[11] F. S. Atiya, A. M. Soliman, and T. N. Saadawi, "Active RC Nominum Phase Network Using the DVCCS/DVCVS," Proc. IEEE, vol. 65, pp. 1606-1607, Nov. 1977.

[12] A. M. Soliman, "A Grounded Inductance Simulation Using the DVCCS/DVCVS," Proc. IEEE, vol. 66, pp. 1089-1091, Sept.1978.

[13] R. Nandi, "New Ideal Active Inductance and FrequencyDependent Negative Resistance Using DVCCS/DVCVS: Applications in Sinusoidal-Oscillator Realization," Electron. Lett., vol. 14, pp. 551-553, Aug. 1978.

[14] I. M. Filanovsky and K. A. Stromsmoe, "More ActiveRC Filters Using DVCCS/DVCVS," Electron. Lett., vol. 15 pp. 466-467, Aug. 1979.

[15] D. Patranabis and A. Paul, "Floating Ideal Inductor with One DVCCS," Electron. Lett., vol. 15, pp. 545-546, Aug. 1979.

[16] T. Deliyannis, "Active RC Filters Using an Operational Transconductance Amplifier and an Operational Amplifier," Int. J. Circuit Theory Appl., vol. 8, pp. 3954, Jan. 1980.

[17] A. Urbas and J. Osiwski, "High-Frequency Realization of C-OTA Second-Order Active Filters," Proc. IEEE/ISCAS, pp. 1106-1109, 1982.

[18] H. S. Malvar, "Electronically Controlled Active Filters with Operational Transconductance Amplifiers," IEEE Trans. Circuits Syst., vol. CAS-29, pp. 333-336, May 1982.

[19] R. L. Geiger and J. Ferrell, "Voltage Controlled Filter Design Using Operational Transconductance
Amplifiers," Proc. IEEE/ISCAS, pp. 594-597, May 1983.

[20] A. R. Saha and R. Nandi, "Integrable Tunable Sinusoid Oscillator Using DVCCS," Electron. Lett., vol. 19, pp. 745-746, Sept. 1983.

[21] G. M. Wierzba and S. Esmeliogiu, "Techniques for Designing Enhanced-Gain-Bandwidth-Product Circuits," Proc. 26th Midwest Symp. on Circuits and Syst., pp. 602-606. Aug. 1983.

[22] R. W. Newcomb and S. T. Liu, "A Voltage Tunable Active-R Filter," Proc. IEEE/ISCAS, pp. 409-412, May 1984.

[23] J. Hoyle and E. Sánchez-Sinencio, "Sinusoidal Quadrature OTA Oscillators," Proc. 27th Midwest Symp. on Circuits and Syst., June 1984.

[24] H. S. Malvar, "Electronically Controlled Active Active$\mathrm{C}$ Filters and Equalizers with Operational Transconductance Amplifiers," IEEE Trans. Circuits Syst., vol. CAS-31, pp. 645-649, July 1984.

[25] F. Krummenacher, "High-Voltage Gain CMOS OTA for Micropower SC Filters," Electron. Lett., vol. 17, pp. 160-162, Feb. 1981.

[26] M. G. Degrauwe, J. Rijmenants, E. A. Vittoz, and J. deMan, "Adaptive Biasing CMOS Amplifiers," IEEE J. Solid-State Circuits, vol. SC-17, pp. 522-528, June 1982.

[27] K. D. Peterson and R. L. Geiger, "CMOS OTA Structures with Improved Linearity," Proc. 27th Midwest Symp. on Circuits and Syst., June 1984.

[28] A. Nedungadi and T. R. Viswanathan, "Design of Linear CMOS Transconductance Elements," IEEE Trans. on Circuits and Syst., vol. CAS-31, pp. 891-894, Oct. 1984.

[29] K. S. Tan and P. R. Gray, "Fully Integrated Analog Filters Using Bipolar-JFET Technology," IEEE J. Solid-State Circuits, vol. SC-12, pp. 814-821, Dec. 1978.

[30] A. P. Nedungadi and P. E. Allen, "A CMOS Integrator for Continuous-Time Monolithic Filters," Proc. IEEE/ISCAS, vol. 2, pp. 932-935, May 1984.

[31] G. Tröster and W. Langheinrich, "Monolithic Continuous Time Analogue Filters in NMOS Technology," Proc. IEEE/ISCAS, vol. 2, pp. 924-927, May 1984.

[32] H. Khorramabadi and P. R. Gray "High-Frequency CMOS

Continuous-Time Filters," Proc. IEEE/ISCAS, vol. 3, pp. 1498-1501, May 1984.

[33] K. Fukahori, "A Bipolar Voltage-Controlled Turnable Filter," IEEE J. Solid-State Circuits, vol. SC-16, pp. 729-737, Dec. 1981.

[34] K. W. Moulding, "Fully Integrated Selectivity at High Frequency Using Gyrators," IEEE Trans. Broadcast. Telev, Reg., vol. BTR-19, pp. 176-179, Aug. 1973.

[35] H. 0. Voorman and A. Biesheuvel, "An Electronic Gyrator," IEEE J. Solid-State Circuits, vol. SC-7 pp, 469-474, Dec. 1972.

[36] K. W. Moulding and P. J. Ranking, "Experience with High-Frequency Gyrator Filters Including a New Video 
Delay-Line IC," Proc. 6th European Conf. on Circuit Theory and Design, pp. 95-98, Sept. 1983.

[37] J. 0. Voorman, W. H. A. Brüls, and J. P. Barth, "Bipolar Integration of Analog Gyrator and Laguerre Type Filters (Transconductance-Capacitor Filters)," Proc. 6th European Conf. on Circuit Theory and Design, pp. 108-113, Sept. 1983.

[38] J. 0. Voorman, W. H. A. Brills, and P. J. Barth, "Integration of Analog Filters in a Bipolar Process," IEEE J. of Solid-State Circuits, vol. SC-17, pp. 713722, Aug. 1982.

[39] K. W. Moulding, J. R. Quarterly, P. J. Rankin, R. S. Thompson, and G. A. Wilson, "Gyrator Video Filter IC with Automatic Tuning," IEEE J. of Solid-State Circuits, vol. SC-15, pp. 963-968, Dec. 1980.

[40] V. W. Burgger, B. J. Hosticka, and G. S. Moschytz, "A Comparison of Semiconductor Controlled Sources for the Design of Active RC Impedances," Int. J. of Circuit Theory and Appl., vol. 10, pp. 27-42, 1982.

Randall L. Geiger was born in Lexington, Nebraska, on May 17, 1949. He received the B.S. degree in electrical engineering and the M.S. degree in mathematics from the University of Nebraska, Lincoln, in 1972 and 1973, respectively. He received the Ph.D. degree in electrical engineering from Colorado State University, Fort Collins, in 1977.

In 1977, Dr. Geiger joined the Faculty of the Department of Electrical Engineering at Texas A\&M University, College Station, and currently holds the rank of Associate Professor. His present research is in the areas of integrated circuit design and active circuits. He received the Meril B. Reed Best Paper Award at the 1982 Midwest Symposium on Circuits and Systems, served as Conference Chairman at the 1983 UGIM conference, and is currently serving as an Associate Editor for the IEEE Transactions for Circuits and Systems.

Dr. Geiger is a member of Eta Kappa Nu, Sigma Xi, Pi Mu Epsilon, and Sigma Tau; he is also a Senior Member of the IEEE.

Edgar Sánchez-Sinencio was born in Mexico City, Mexico, on October 27, 1944. He received the degree in communications and electronic engineering (professional degree) from the National Polytechnic Institute of Mexico, Mexico City; the M.S.E.E. degree from Stanford University, Califomia; and the Ph.D. degree from the University of Illinois at Champaign-Urbana, in 1966, 1970, and 1973, respectively.

During his graduate studies, he was awarded with fellowships from the United Nations Educational, Scientific, and Cultural Organization; the Mexican Atomic Energy Commission; and the Consejo Nacional de Ciencia y Tecnologia of Mexico. From January 1965 to March 1967, he worked with the Mexican Atomic Energy Commission, as a Design Engineer.

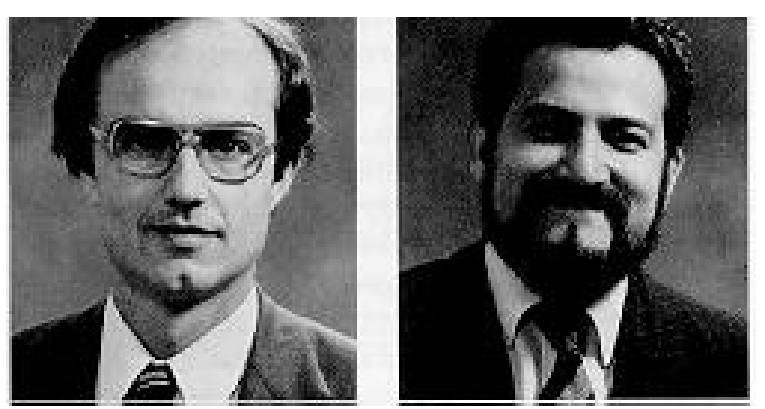

In April 1967, he joined the Petroleum Institute of Mexico, where he was associated with the design of instrumentation equipment until August 1967. He worked as a Research Assistant at the Coordinated Science Laboratory at the University of Illinois from September 1971 to August 1973.

In 1974, Dr. Sánchez-Sinencio held an industrial postdoctoral position with the Central Research Laboratories, Nippon Electric Company, Ltd., Kawasaki, Japan. From 1976 to 1983, he was the Head of the Department of Electronics at the Instituto Nacional de Astrofisica, Optica y Electrónica (INAOE), Puebla, Mexico. Dr. Sánchez-Sinencio was a Visiting Professor in the Department of Electrical Engineering at Texas A\&M University during the academic years of 1979-1980 and 1983-1984, where he is currently a Professor. He was the General Chairman of the 1983 26th Midwest Symposium on Circuits and Systems. He is the coauthor of the book Switched-Capacitor Circuits (Van Nostrand-Reinhold, 1984). Dr. Sánchez-Sinencio's present interests are in the areas of active filter design, solid-state circuits, and computer-aided circuit design. $\mathrm{He}$ is a Senior Member of the IEEE. 